\title{
Robot Orientation with Histograms on MSL
}

\author{
Fernando Ribeiro, Gil Lopes, Bruno Pereira, João Silva, Paulo Ribeiro, \\ João Costa, Sérgio Silva, João Rodrigues, and Paulo Trigueiros \\ Industrial Electronics Department, Univ. of Minho, \\ Campus de Azurém, 4800-058 Guimarães, Portugal \\ \{gil, fernando\} @dei.uminho.pt, \\ \{brunomiguel1987, jsilva86, paulorogeriocp, \\ joaovazcosta, sergio.fil, pedro50073\} egmail.com, \\ paulo@trigueiros.com \\ http: //www.minhoteam.org
}

\begin{abstract}
One of the most important tasks on robot soccer is localization. The team robots should self-localize on the 18 x 12 meters soccer field. Since a few years ago the soccer field has increased and the corner posts were removed and that increased the localization task complexity. One important aspect to take care for a proper localization is to find out the robot orientation. This paper proposes a new technique to calculate the robot orientation. The proposed method consists of using a histogram of white-green transitions (to detect the lines on the field) to know the robot orientation. This technique does not take much computational time and proves to be very reliable.
\end{abstract}

Keywords: Robot Orientation, Histogram, Robot Localization, Middle Size League.

\section{Introduction}

RoboCup consists of a scientific challenge of Artificial Intelligence, whose main objective is to build a team of robots to play football against a human team. The scheduled year for that is 2050. Since 1997, annually new challenges have been added in order to improve the quality of game on RoboCup. Some of these challenges were the field size increase and the removal of the corner landmarks.

In order to play soccer each robot uses a vision system to gather information of the surrounding area, the robot must be able to move, pass and kick the ball, collaborate with team members, obey to referee commands, etc. Almost all robot decisions are dependent on its localization. Therefore, one of the main problems in autonomous football robots can be considered self-localization.

The recent implemented changes on the field are responsible for the blossom of new ways for self-localization. Before removing the corner landmarks almost all localization methods relied on those cylinders. The increasing of the soccer field size also encourages new localization methods, since most robots can only see around about 4 or 5 meters radius while the field is $18 \times 12$ meters. 
One of the most used algorithms for self-localization is Monte Carlo [8] [1] [5]. Since Monte Carlo is very used, some improvements on it had been proposed [3] [7].

However, rather than proposing a new localization algorithm, this paper proposes a new way of sorting out one step of the localization task, namely the orientation. The proposed approach uses histograms of the transitions between white lines and the green colour on soccer field.

The histogram of the transitions is used to find out the robot orientation. One important aspect to take into account is the time required for the localization task; the better method is dependent on the compromise between the shortest time and the highest rate of correct position.

This paper is organized as follows. In Section 2, the localization state of the art is described. In Section 3, the proposed approach which uses histogram is presented. Section 4 describes the methodology used. Section 5 shows up some results. Section 6 has a discussion about existing localization methods. Finally, Section 7 presents the conclusions and directions for further work.

\section{Background}

The team robots moving strategy requires localization of all robots of the team, to find the goals to kick the ball. These reasons make the localization one of the main problems on football robots. Before removing the surrounding walls, the landmarks, and the corners and increasing the size of the football field, the localization was not so difficult, but nowadays it is a hard task. In [10] one can read a discussion about the effect on localization after the removal of walls, as it talks about the problem on localization due to field size increase.

At that time, the sensors most used were laser range finder, odometry and vision system. However, the laser ranger was more used before removing the walls and landmarks.

With respect to odometry the problem is the obtained error, since the reading of encoder is not feasible with such slipping wheels like omnidirectional wheels. In order to avoid problems with odometry some works propose a way to fix the reading of the encoders [4]. The sensor still in use, nowadays, is the vision system [7]. The advantage of vision systems is the possibility of observing $360^{\circ}$.

The most used algorithm to self-localization is Monte Carlo [8] [1] [5]. The approach is Sequential Monte Carlo or also called Particle Filter. Since this method was broadly used some improvements had been proposed. This work [3] proposes a method to the number of samples to be adaptive on Monte Carlo. Already on [7] the improvement is to use Genetic Algorithm, the evolution operators to generate points on regions with high post density.

The proposed method in [6] is also very used [9] [2]. It transforms the localization in an optimization problem. The robot position is the minimum error, i.e. the minimum of the function. It uses the Kalman Filter to improve the solution, i.e. to make it smoother. Work [6] has a comparison with Monte Carlo. 


\section{Histogram Approach}

The robot location is composed by [x y $\theta$ ], being $\theta$ the robot orientation. The proposed approach uses histograms to identify the orientation. These histograms count the transitions between white lines and the green colour on the soccer field, and these transitions are found by axial searching lines and by radial searching lines. Histograms are built in two directions; a vertical one and an horizontal one.

Figure 1 shows an example of the system. The image on the centre shows what the robot sees, the green field with the white lines, with axial lines and radial lines, and the transitions represented by dots. The figure on the left shows the transitions represented by white dots, the horizontal histogram (underneath) represented by straight lines and the vertical histogram (on the right side) represented by straight lines, of the grabbed image. The figure on the right represents the same information as on the left part of the image, but with the proper rotation after calculation (transition are now parallel to the $\mathrm{X}-\mathrm{Y}$ axis).

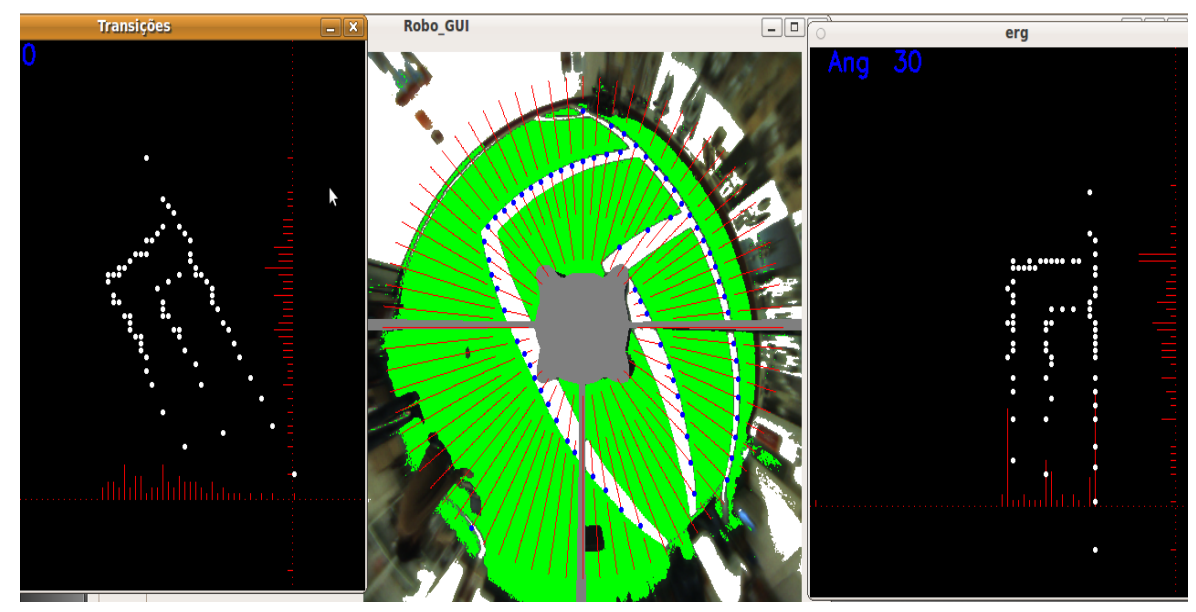

Fig. 1. Screen capture of the real image with the transition white/green dots (middle), the conversion to real distance (left), the rotation and angle displacement finding (right)

The proposed approach in order to calculate the orientation of the robot is: Rotate the histogram (first part) on the interval $\theta-40^{\circ}$ until $\theta+40^{\circ}, \theta$ is the last known orientation, and verify the histogram maximum value (peak value) for each rotation. Each histogram maximum value, sum of maximum vertical and maximum horizontal, and the corresponding angle should be saved. The angle in which the highest value achieved by summing the horizontal and the vertical histogram values, corresponds to the new orientation of the robot. The 40 was chosen because on one frame the robot can only rotate this value, at most.

On Figure 1 it can be seen that a larger peak value is achieved when the transitions are all aligned (third part), therefore, rotating the histogram (first part) some degrees, in this case $30^{\circ}$, the maximum peak is achieved. 
The decision to use the maximum sum of horizontal and vertical histogram values was because in some cases one or two histograms can have more than one maximum, and therefore, more than one solution. The final solution is evaluated with these two histograms to fuse the information but some other approaches could be used, like the Mean of these values or considering only the greater value of both. However, the adopted solution was the maximum sum of them, and when in practice it proves much more reliable. Sometimes there are many similar histogram peak values. For example, on Figure 2, one can see on interval 19 to 23, 29 to 32, and 36 to 40 for vertical histogram. In order to decide the right angle, the sum between horizontal and vertical histograms is used, therefore, removing the ambiguity. In this case the angle is $30^{\circ}$.

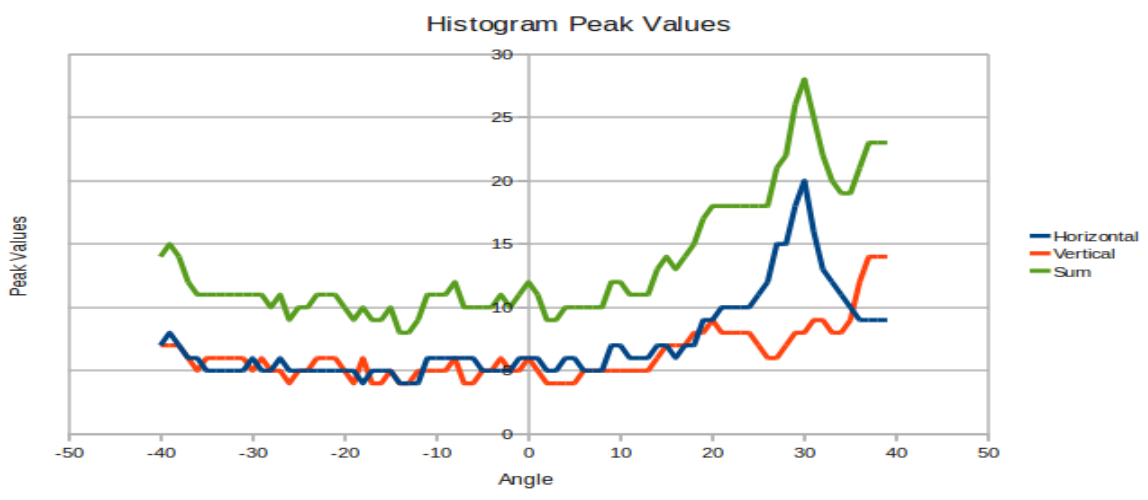

Fig. 2. Histogram peak values of the given example where a maxima was reached at $30^{\circ}$ on the horizontal and hence the Sum histogram

\section{Experiments}

In order to validate the method, 5 experiments were carried out, the robot was placed in 5 different places and with different orientations on the soccer field. Figure 3 shows the locations where the robots were placed to carry out these experiments. The locations chosen were selected in paces with many lines visible, far away from any line, near the corner, near the side line, and near a non-straight line. Due to space restrictions, in the laboratory there is only one half of the soccer field, and therefore, the experiments were carried out only on one side. The size of the half soccer field, in our laboratory, is 8.24 meters width by 6.07 meters length.

The locations and orientations chosen have the aim to validate the method, the transitions occur in higher quantities than in others. The approach in order to compare the angle's results of the histogram used trigonometric methods.

\section{$5 \quad$ Results}

The software was developed using C++ language, QtCreator IDE and Ubuntu linux operating system. The computer used was a conventional notebook with a $2.2 \mathrm{GHz}$ 


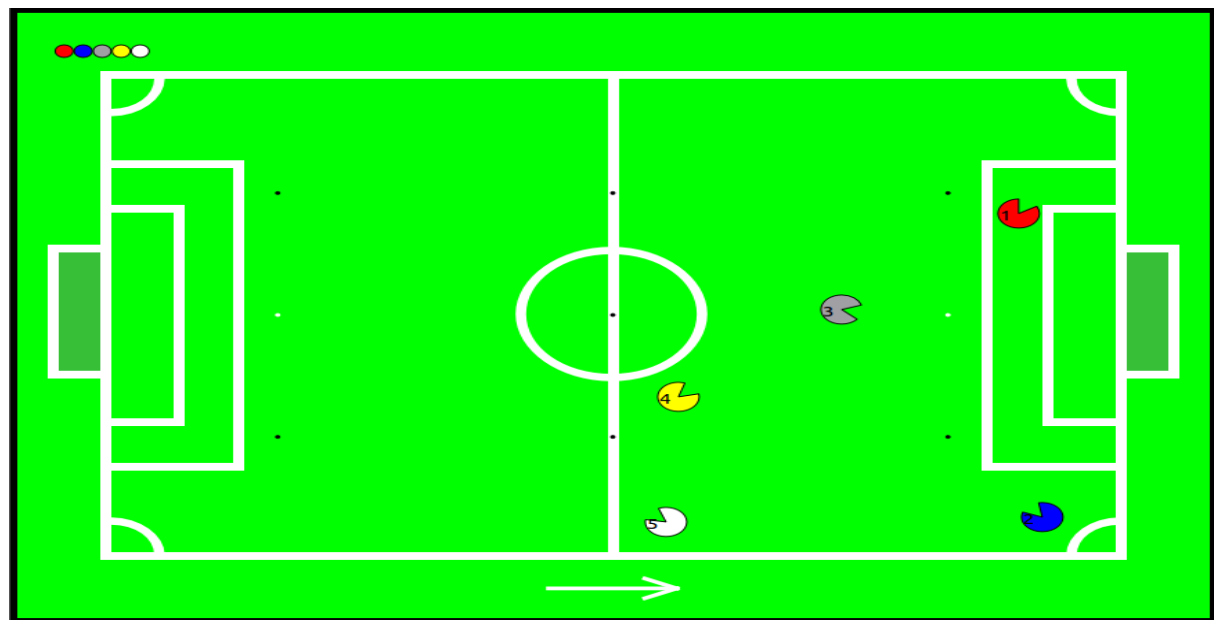

Fig. 3. Conducted experiments with the robot field positions used on the experiments

Core 2 Duo processor. The experiments were: First with $30^{\circ}$, Second with $-40^{\circ}$, Third with $102^{\circ}$, Fourth with $48^{\circ}$ and Fifth with $-51^{\circ}$. In all cases the error obtained was below $2^{\circ}$, which is very acceptable considering that robot moves very fast, and the calculation is carried out every frame. A negative rotation is counter-clockwise (see experiments second and fifth).

Figure 4 shows the result of the third experiment, and Figure 5 shows a graphical representation of the vertical histogram, the horizontal histogram and the sum of these two histograms. In this case, the robot was heading to $120^{\circ}$, and since the search starts $40^{\circ}$ before and ends $40^{\circ}$, it started on $80^{\circ}$ and ended on $160^{\circ}$. It found the solution on $-18^{\circ}$, which corresponds to $120-18$ or in other words the robot is heading to $102^{\circ}$.

Once again several peaks can be seen on the histograms. On Figure 5, two peaks near each other can be seen on the horizontal histogram, while the vertical is almost constant. However, the peaks are always near.

Figure 6 shows the result of the fifth experiment, and Figure 7 shows the graphical representation of the two histograms and its sum. The robot was heading to angle $-20^{\circ}$. Figure 7 shows the maximum value of the vertical histogram was not the right angle, since the right angle was reached because the horizontal overcomes the vertical.

\section{Discussion}

The proposed method is a new way for robot orientation. It only uses the histograms of transitions between white lines and the green field colour on robotic football fields. These transitions are detected by axial and radial lines. Two histograms are used, one horizontal and one vertical, and to fuse these two histogram a sum of maximum values of each is adopted. 


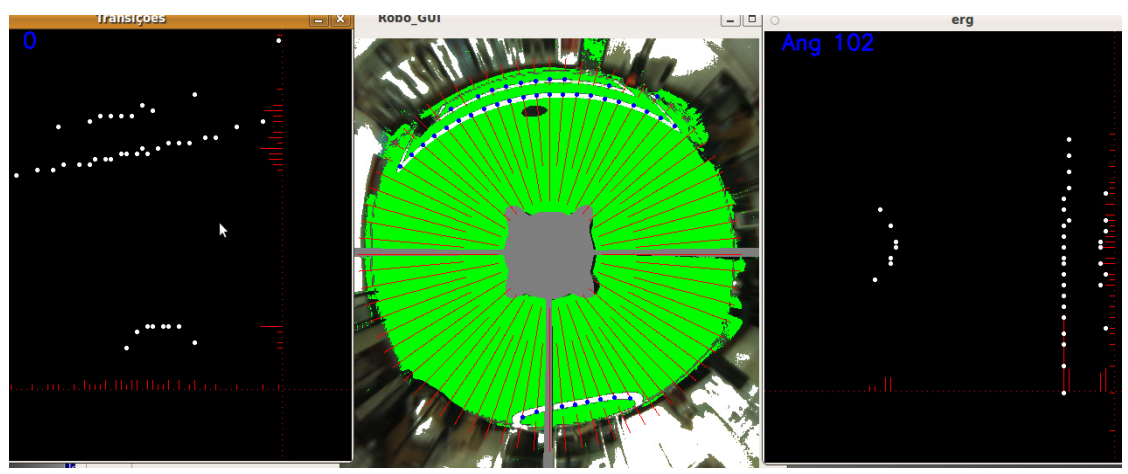

Fig. 4. Screen capture of the real image with the transition white/green dots (middle), the conversion to real distance (left), the rotation and angle displacement finding (right), all of experiment 3

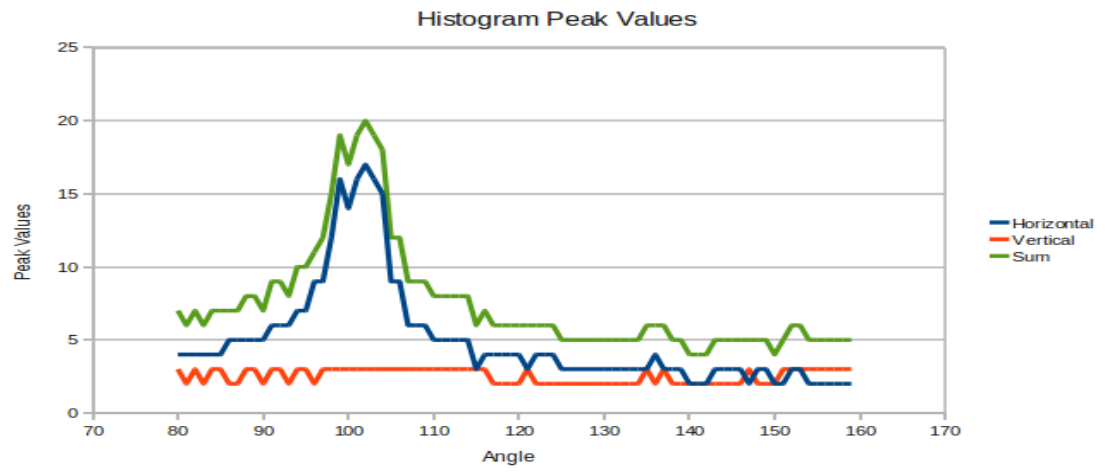

Fig. 5. Histogram peak values of experiment 3 where a maxima was reached at $102^{\circ}$ on the horizontal and hence the Sum histogram

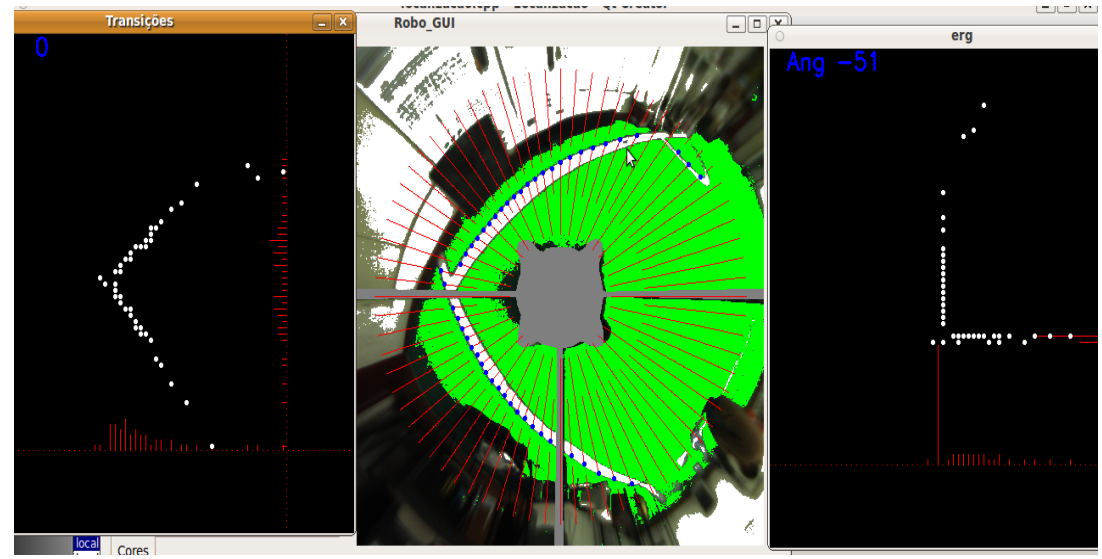

Fig. 6. Screen capture of the real image with the transition white/green dots (middle), the conversion to real distance (left), the rotation and angle dis-placement finding (right), all of experiment 5 
The processing time spent for angle detection is only 4 milliseconds. Another advantage is its easiness to use it in other localization methods, because it only needs the transitions, and some methods use these transitions.

A sum of the histograms to remove ambiguity demonstrated to be reliable, because it can calculate the angle when the maximum peaks of the histograms are situated on different intervals.

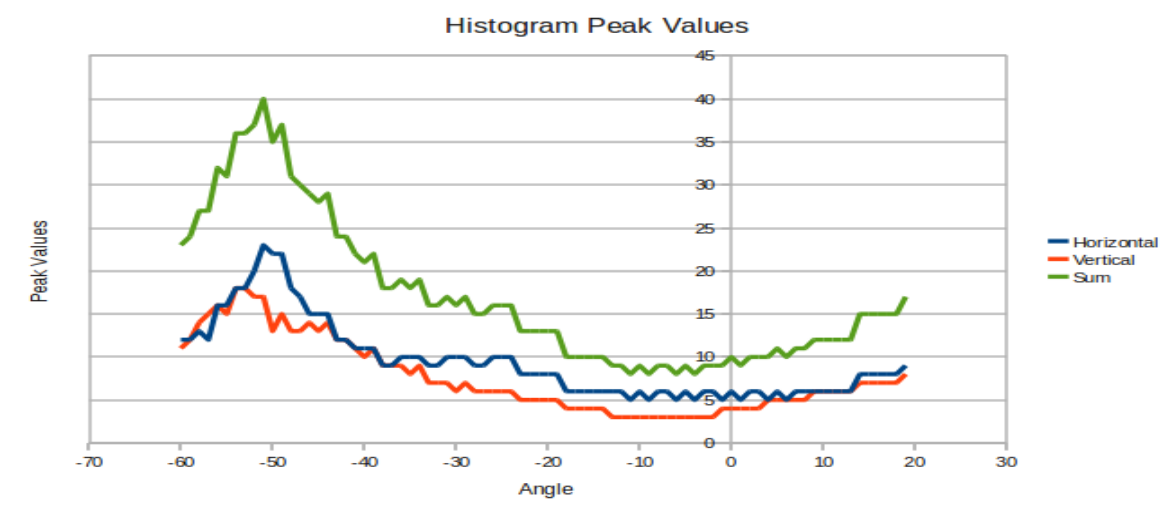

Fig. 7. Histogram peak values of experiment 3 where a maxima was reached at $-51^{\circ}$ on the horizontal and hence the Sum histogram

\section{Conclusions}

The localization is an important task on the robotic football environment and one element present on this task is orientation. This paper presents a new way to calculate the robot orientation using only histograms. The approach to build this is the transitions between white lines and the green colour on soccer field. The transitions are found using axial and radial searching lines. There is a vertical and a horizontal histogram.

The proposed method only requires 4 milliseconds to calculate the correct robot orientation. Therefore, it can be used on real football environment without penalizing others computing task, like robot planning and vision systems, allowing the robot's main computer to process between 25 and 30 frames per second.

Acknowledgements. The authors wish to thank all members of the Laboratorio de Automacao e Robotica, at University of Minho, Guimaraes. Also special thanks to the ALGORITMI Research Centre for the opportunity to develop this research. 


\section{References}

1. Beck, D., Niemueller, T.: Allemaniacs team description paper: Robocup (2009)

2. Hafner, R., Lange, S., Riedmiller, M., Welker, S.: Brainstormers tribots team description paper: RoboCup (2009)

3. Heinemann, P., Haase, J., Zell, A.: A Novel Approach to Efficient Monte-Carlo Localization in RoboCup. In: Lakemeyer, G., Sklar, E., Sorrenti, D.G., Takahashi, T. (eds.) RoboCup 2006. LNCS (LNAI), vol. 4434, pp. 322-329. Springer, Heidelberg (2007)

4. Hundelshausen, F.V., Schreiber, M., Wiesel, F., Liers, A., Rojas, R.,, M.: A force field pattern matching method for mobile robots. Tech. rep. (2003)

5. Komoriya, Y., Hashimoto, R., Miyoshi, K., Ogawa, Y., Sakakibara, D., Sawada, J., Tahara, K., Fukushima, H., Mitani, K., Yamakage, R., Asano, Y., Demura, K.: Winkit team description paper: RoboCup (2009)

6. Lauer, M., Lange, S., Riedmiller, M.: Calculating the Perfect Match: An Efficient and Accurate Approach for Robot Self-Localization. In: Bredenfeld, A., Jacoff, A., Noda, I., Takahashi, Y. (eds.) RoboCup 2005. LNCS (LNAI), vol. 4020, pp. 142-153. Springer, Heidelberg (2006)

7. Luo, R., Min, H.: A new omni-vision based self-localization method for soccer robot. In: Proceedings of the 2009 WRI World Congress on Software Engineering, WCSE 2009, vol. 1, pp. 126-130. IEEE Computer Society, Washington, DC (2009)

8. Nassiraei, A.A., Kitazumi, Y., Ishida, S., Toriyama, H., Ono, H., Takenaka, K., Shinpuku, N., Takaki, M., Fukunaga, Y., Yamada, K., Takemura, Y., Godler, I., Ishii1, K., Miyamoto, H.: Hibikino-musashi team description paper: RoboCup (2010)

9. Su, S., Gu, Z., Chen, X., Dong, L., Huang, Y., Song, X., Wu, L.: Endeavor team description paper: RoboCup (2010)

10. Utz, H., Neubeck, A., Mayer, G., Kraetzschmar, G.K.: Improving Vision-Based SelfLocalization. In: Kaminka, G.A., Lima, P.U., Rojas, R. (eds.) RoboCup 2002. LNCS (LNAI), vol. 2752, pp. 25-40. Springer, Heidelberg (2003) 